\title{
Opportunities with Decay-At-Rest Neutrinos From Decay-In-Flight Neutrino Beams
}

\author{
Christopher Grant* \\ Boston University \\ E-mail: cpgrantebu. edu
}

The decay-at-rest neutrino flux has been investigated at the existing Fermilab NuMI beam facility. Decay-at-rest neutrino production in NuMI is found to be roughly equivalent per megawatt to that of spallation facilities, and is concentrated in the facility's target hall and beam stop regions. Special attention is focused on the opportunity to measure neutrino-argon reactions in the energy range of stellar collapse. Interaction rates in a 5 ton liquid argon detector at existing and hypothetical locations near the NuMI target hall are reported. The physics implications and experimental challenges of such a measurement are also discussed.

38th International Conference on High Energy Physics 3-10 August 2016

Chicago, USA

${ }^{*}$ Speaker. 


\section{Introduction}

The J-PARC Neutrino Experimental Facility [1], the CNGS beam at CERN [2], and the Booster [3] and Main Injector [4] beams at Fermilab have served as primary neutrino physics facilities producing decay-in-flight (DIF) neutrinos and antineutrinos in the $\sim 0.5-50 \mathrm{GeV}$ range. Detectors situated in the paths of these beams have produced an impressive array of physics including measurement of neutrino oscillations $[5,6,7]$, searches for sterile neutrinos [8], and new insights into nuclear/nucleon structure [9].

While producing a high-energy neutrino beam, these facilities also produce a far-more-copious flux of lower-energy neutrinos from the decay-at-rest (DAR) of pions, kaons, and muons. A large majority of charged mesons produced by proton beams are not focused by the horns and instead DAR in or near the target. Of the horn-focused component, many mesons and product muons reach the end of the beam drift region without decaying, instead terminating via DAR in large beam stops. The resultant fluxes of DAR $v_{e}, v_{\mu}$, and $\bar{v}_{\mu}$ are similar in character to those produced at nuclear physics facilities primarily dedicated to the production of spallation neutrons [10].

Pion and muon DAR neutrinos in the $10-50 \mathrm{MeV}$ range are ideal for low-energy neutrino cross-section studies. The observation of $\mu^{+}$DAR $v_{e}$ in a liquid argon detector would elucidate the range of complex final states produced by supernova and solar neutrinos in future large underground experiments, such as DUNE [11]. With this in mind, the DAR neutrino reaction rates for a 5 ton liquid argon detector positioned near the NuMI target hall have been explored in detail.

\section{Method and Results}

The calculation of neutrino production by the NuMI beam was performed using the NUMIX collaboration's G4NuMI software, a pure GEANT4-based code $[12,13]$ that propagates particles through a realistic geometry of the NuMI beam facility. The geometry includes the target hall containing a graphite target followed by two magnetic focusing horns, all of which is encased in layers of steel and concrete shielding. A $675 \mathrm{~m}$ long, $2 \mathrm{~m}$ diameter steel decay pipe begins at the end of the target hall and is filled with helium gas. A beam dump, called the NuMI hadron absorber, is located at the end of the decay pipe and is made up of a $1.3 \mathrm{~m}$ wide $\times 1.3 \mathrm{~m}$ high $\times 4.75 \mathrm{~m}$ long core containing aluminum blocks and ten steel blocks, surrounded by additional steel and concrete. The entire beam line is centered along one axis in a cylindrical world volume of rock $1200 \mathrm{~m}$ long and $25 \mathrm{~m}$ in diameter extending roughly $400 \mathrm{~m}$ past the absorber end. Further description of the NuMI facility can be found in Ref. [4].

For DAR, the primary neutrino production channels are $K^{+} \rightarrow v_{\mu} \mu^{+}, \pi^{+} \rightarrow v_{\mu} \mu^{+}$, and $\mu^{+} \rightarrow$ $\bar{v}_{\mu} v_{e} e^{+}$. Other contributions also come from $\mu^{-} p \rightarrow n v_{\mu}$ (nuclear capture) and $\mu^{-} \rightarrow v_{\mu} \bar{v}_{e} e^{-}$ (decay in orbit around the atomic nucleus). Fluxes incident on $1 \mathrm{~m}^{2}$ areas are calculated at predefined locations in $(x, y, z)$ coordinates. To good approximation, all DAR neutrinos are emitted isotropically.

The rates of the most significant contributions are found to be $0.5 K^{+}, 11.0 \pi^{+}, 27.3 \mu^{+}$, and $1.9 \mu^{-}$DAR $v$ /proton. These totals are a factor of $\sim 200-400$ higher per proton than lower-energy spallation $v$ sources $[14,15]$, compensating for the lower overall protons on target produced in DIF facilities. When production is compared per MW beam power the DAR production at DIF 

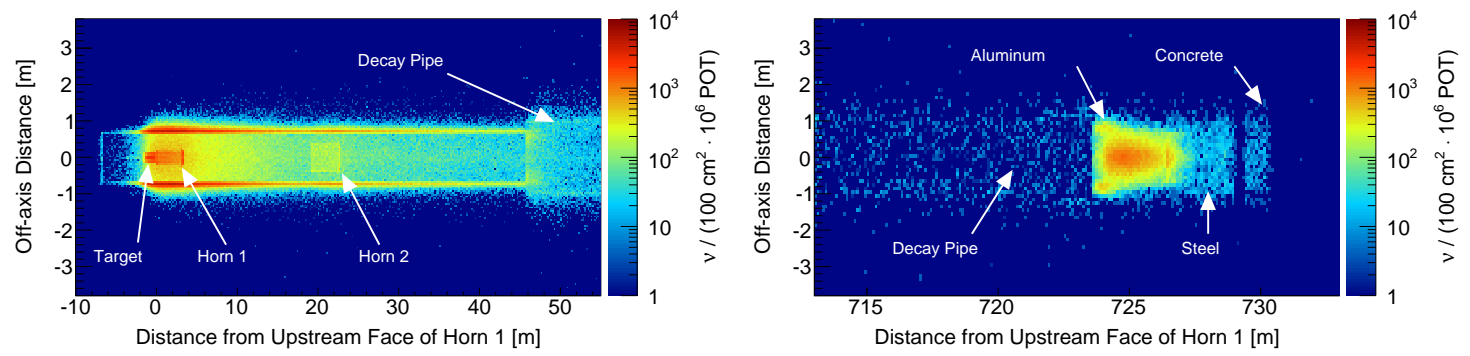

Figure 1: Neutrino origin distributions inside the NuMI target hall (top) and hadron absorber (bottom), as viewed from above.

facilities to be roughly equal to spallation facilities. This same general equivalence per MW also applies between the $700 \mathrm{~kW}$ NuMI and $32 \mathrm{~kW}$ Booster beamlines, indicating roughly an order of magnitude lower DAR neutrino production at the Booster.

The flux from DAR neutrinos is highest in two locations: the target hall and the hadron absorber. This can be seen in Fig. 1 where the distribution of stopped neutrino parents is shown in the 2D plane, viewed from above. Neutrino production is highest in the target hall: $80 \%$ of $v$ are produced within a $2 \mathrm{~m} \times 2 \mathrm{~m} \times 60 \mathrm{~m}$ rectangular box surrounding these target and horn areas. Of this majority, only $4 \%$ and $6 \%$ are produced directly within the target and horns, respectively, highlighting the important role of surrounding shielding material in low-energy neutrino production. On the other end of the decay pipe, a smaller but substantial $12 \%$ of DAR flux is produced within a $3 \mathrm{~m} \times 3 \mathrm{~m}$ x $4 \mathrm{~m}$ rectangular box encompassing the hadron absorber core.

The neutrino flux below $100 \mathrm{MeV}$ is shown in Fig. 2 at an example distance of $25 \mathrm{~m}$ offaxis directly to the side of the target. The flux below $53 \mathrm{MeV}$ is almost entirely from $\pi^{+}$and $\mu^{+}$ DAR with minor (4\%) DIF contamination. The two-body $\pi^{+}$decay produces a mono-energetic $29.8 \mathrm{MeV} v_{\mu}$, and the three-body $\mu^{+}$decay produces a continuous spectrum of $v_{e}$ and $\bar{v}_{\mu}$ up to 53 $\mathrm{MeV}$. The shoulder extending up to $100 \mathrm{MeV}$ is a byproduct of $\mu^{-}$capture on the nuclei of material in the target hall. The inset of Fig. 2 shows the total flux from all flavors out to $250 \mathrm{MeV}$ to make visible the flux of mono-energetic $235.5 \mathrm{MeV} v_{\mu}$ from the two-body $K^{+}$DAR. DIF contamination to $K^{+}$DAR, $1 \%$ between 230 and $240 \mathrm{MeV}$, is also low. Spectral features from the absorber are similar, with $2 \%$ DIF contamination below $53 \mathrm{MeV}$ and a higher $13 \%$ for $\mathrm{K}^{+}$DAR between 230 and $240 \mathrm{MeV}$.

\section{Physics Opportunities}

In a liquid argon detector, the dominant process for neutrino detection below $100 \mathrm{MeV}$ is through the charged-current (CC) reaction: $v_{e}+{ }^{40} \mathrm{Ar} \rightarrow{ }^{40} \mathrm{~K}^{*}+e^{-}$. The flavor of the incoming neutrino is determined by the tagging the outgoing electron track (in the case of a Liquid Argon Time Projection Chamber). The energy of the incoming neutrino is not so easily inferred due to a strong nuclear structure dependence. Unlike inverse beta decay on hydrogen, which produces a single final state, the reaction on argon has several complexities: 


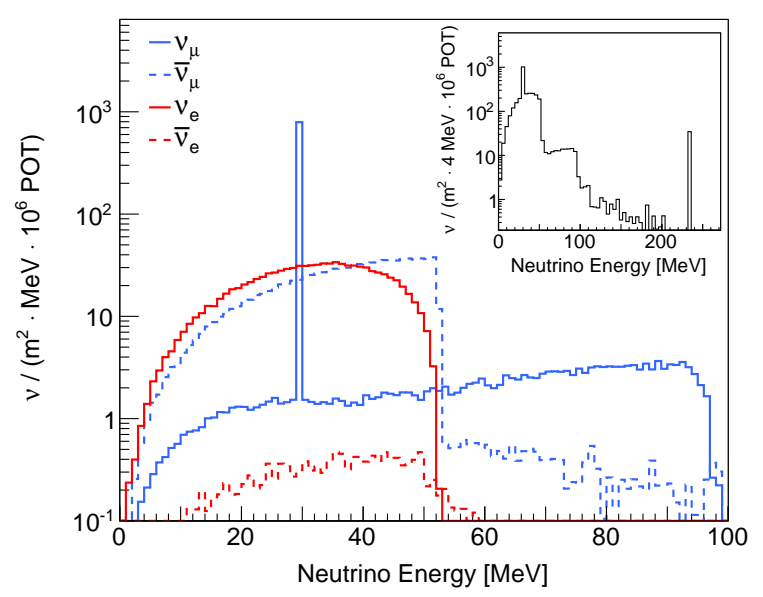

Figure 2: Neutrino flux at $25 \mathrm{~m}$ distance from the NuMI target with an inset showing the total flux for all flavors.

- The ground state $\left(J^{P}=0^{+}\right)$to ground state $\left(J^{P}=4^{-}\right)$transition is third forbidden, thereby forcing a transition to one of the 20 (or more) allowed excited states. Excited states will produce a combination of electrons and gammas that need to be reconstructed in order to determine the incoming neutrino energy.

- The thresholds and transition strengths to most of the allowed excited states are poorly known $[16,17,18]$. Furthermore, more than half of the excited states have no gamma deexcitation data.

- Nuclear fragmentation occurs at an excitation energy of $\sim 12 \mathrm{MeV}$, meaning that many of the final states will contain baryons (most likely neutrons and protons). The number of baryons produced and uncertainties in neutron transport in argon will complicate neutrino energy reconstruction.

Determining the neutrino energy requires detection of all de-excitation particles emitted from ${ }^{40} \mathrm{~K}^{*}$. An extensive effort has been made to gather all available measurements and theoretical calculations of transition intensities to the allowed excited states of ${ }^{40} \mathrm{~K}$. These data have been combined into a neutrino event generating software called MARLEY, which stands for Model of Argon Reaction Low Energy Yields [19]. Predictions from other sub-dominant reactions $\left(\bar{v}_{e}+{ }^{40} \mathrm{Ar} \rightarrow{ }^{40} \mathrm{Cl}^{*}+\right.$ $e^{+}$and $v_{x}+{ }^{40} \mathrm{Ar} \rightarrow{ }^{40} \mathrm{Ar}^{*}+v_{x}$ ) require further development of MARLEY, but estimates of the total cross-section exist [20]. A measurement of the neutrino-argon cross-section as a function of incoming neutrino energy would provide a more realistic inputs for this event generating software, thereby providing better sensitivity estimates for DUNE.

For comparison, the reaction rates have been calculated for a 5-ton liquid argon detector at two locations: the closest location at 15 meters distance and a farther location 25 meters from the target. The results of the rate calculations are reported in Table 1 . The optimal detector location will depend not only on the reaction rates, but also the rate of particle interactions from beam-induced backgrounds in any particular location. 


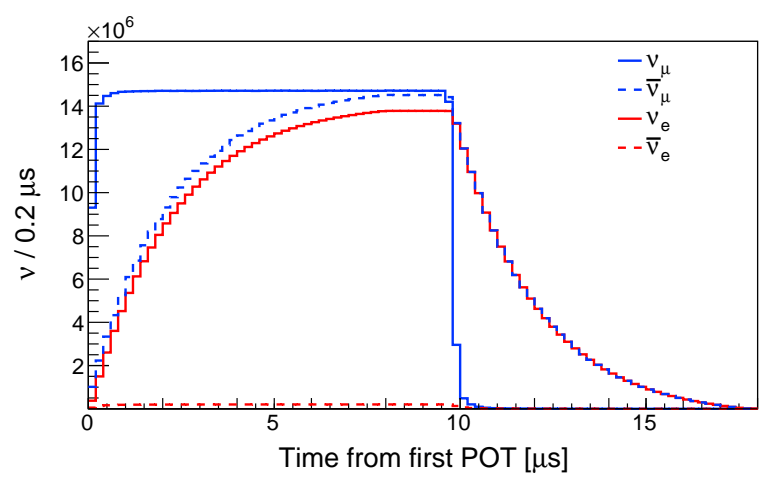

Figure 3: Time profile of neutrino production in the target hall during and after the $10 \mu$ s NuMI beam spill.

Further investigation into backgrounds is an essential component to the low-energy neutrino cross-section measurement mention here. Currently, there are no estimates of backgrounds in this area. Despite inherent suppression from the duty factor of Fermilab's DIF beams, cosmic-induced backgrounds may pose a challenge in the detection of $<100 \mathrm{MeV}$ neutrinos in liquid argon detectors near the surface. The flavor composition near the target varies during a $10 \mu \mathrm{s}$ NuMI spill due to differing meson and $\mu$ decay times, as shown in Fig. 3. A detector utilizing nanosecond timing resolution from its light collection system can use this time structure to select specific neutrino flavors. As an example, for the 25 meter location, by cutting all events in the first $10.4 \mu$ s of the beam spill, the total $v_{\mu}$ contribution to detected event rates below $53 \mathrm{MeV}$ can be reduced by three orders of magnitude with less than one order of magnitude loss in $v_{e}$ signal statistics. It should be noted that, with respect to timing separation cuts, spallation facilities are likely to be more efficient due to their shorter beam spills.

Finally, backgrounds may arise from beam-produced particles or neutrino interactions outside the detector that produce activity inside the liquid argon detector. These contributions are difficult to estimate with existing literature or the simulations described here, but are nonetheless important given the proximity of the examined locations to the major neutrino production sites. Beam-related backgrounds can be determined with direct assays at each examined location with neutron and charged particle spectrometers, while $v$-produced 'dirt' backgrounds can be estimated by propagating the neutrino fluxes described here through a full simulation package employing neutrino interaction generators and subsequent propagation of all final state particles.

\begin{tabular}{lcccc}
\hline \hline Location & LAr Mass $[\mathrm{t}]$ & $\mathrm{CC} v_{e}$ & $\mathrm{NC} v_{\mu}$ & $\mathrm{NC} \bar{v}_{\mu}$ \\
\hline $25 \mathrm{~m}$ from target & 5.0 & 1100 & 125 & 400 \\
$15 \mathrm{~m}$ from target & 5.0 & 3000 & 325 & 1100 \\
\hline
\end{tabular}

Table 1: Calculated neutrino reactions per $6 \times 10^{20}$ POT below $53 \mathrm{MeV}$ in the NuMI target hall. 


\section{Conclusions}

The contribution of low-energy neutrinos in the Fermilab NuMI beamline has been investigated as a site for a decay-at-rest neutrino cross-section experiment. The overall levels of lowenergy neutrino production at NuMI to be roughly equal per MW to those produced by morestudied spallation neutron facilities. A modest 5-ton liquid argon detector placed in the vicinity of the NuMI target or absorber could yield thousands of decay-at-rest $v_{e}$ and $v_{\mu}$ detections. Detailed flux studies for future facilities, further investigation of DIF $v$ contamination, and dedicated background assays in NuMI near-target locations, are recommended as next steps in investigating the short- and long-term potential of this class of experiment.

\section{References}

[1] K. Abe et al. (T2K), Phys. Rev. D87, 012001 (2013), arXiv:1211.0469 [hep-ex] .

[2] G. Giacomelli, J.Phys.Conf.Ser. 116, 012004 (2008), 2nd Latin American School on Cosmic Rays and Astrophysics, Puebla, Mexico, 30th August - 8th September 2006, arXiv:physics/0703247 .

[3] A. A. Aguilar-Arevalo et al. (MiniBooNE), Phys. Rev. D79, 072002 (2009a), arXiv:0806.1449 [hep-ex] .

[4] P. Adamson et al., Nucl. Inst. Methods Phys. Res. Sec. A 806, 279 (2016).

[5] K. Abe et al. (T2K), Phys.Rev. D91, 072010 (2015), arXiv:1502.01550 [hep-ex] .

[6] P. Adamson et al. (MINOS), Phys.Rev.Lett. 112, 191801 (2014), arXiv:1403.0867 [hep-ex] .

[7] N. Agafonova et al. (OPERA), Phys. Rev. Lett. 115, 121802 (2015), arXiv:1507.01417 [hep-ex] .

[8] A. A. Aguilar-Arevalo et al. (MiniBooNE), Phys. Rev. Lett. 103, 111801 (2009b), arXiv:0904.1958 [hep-ex] .

[9] G. T. Garvey, D. A. Harris, H. A. Tanaka, R. Tayloe, and G. P. Zeller, Phys. Rept. 580, 1 (2015), arXiv:1412.4294 [hep-ex] .

[10] B. Louis, V. Sandberg, and H. White, (1997), los Alamos Sci. (1997) No. 25 92-115.

[11] C. Adams et al. (LBNE) (2013) arXiv:1307.7335 [hep-ex] .

[12] S. Agostinelli et al., Nucl. Inst. Methods Phys. Res. Sec. A 506, 250 (2003).

[13] J. Allison et al., IEEE Trans. Nucl. Sci. 53, 270 (2006).

[14] M. Elnimir et al. (OscSNS), (2013), 1307.7097.

[15] S. Axani, G. Collin, J. Conrad, M. Shaevitz, J. Spitz, and T. Wongjirad, (2015), arXiv:1506.05811 [physics.ins-det] .

[16] M. Bhattacharya et al., Phys. Rev. C 58, 3677 (1998).

[17] M. Bhattacharya, C. D. Goodman, and A. García, Phys. Rev. C 80, 055501 (2009).

[18] M. Cheoun, E. Ha, and T. Kajino, Eur. Phys. J. A 48, 137 (2012).

[19] S. Gardiner, K. Bilton, C. Grant,E. Pantic, and R. Svoboda, APS Division of Nuclear Physics Meeting Abstracts (2015), http://www.marleygen.org.

[20] I. Gil-Botella, Journal of Physics: Conference Series 308, 012012 (2011). 